\title{
Prevalence of Orthorexia Nervosa in a Sample of Patients Attending Sligo/Leitrim Mental Health Services with a Diagnosis of Eating Disorder
}

Ignazio Graffeo ( $\square$ graffeoignazio@yahoo.it )

Sligo University Hospital https://orcid.org/0000-0002-8360-7662

Mary Harron

Sligo/Leitrim Mental Health Services

Edmond O'Mahony

Sligo/Leitrim Mental Health Services

\section{Research article}

Keywords: Orthorexia Nervosa, Anorexia Nervosa, Eating Disorders, Bulimia Nervosa, OCD, Obsessive Compulsive Disorder.

Posted Date: August 3rd, 2020

DOI: https://doi.org/10.21203/rs.3.rs-48945/v1

License: (c) (i) This work is licensed under a Creative Commons Attribution 4.0 International License. Read Full License

Version of Record: A version of this preprint was published at BJPsych Open on June 1st, 2021. See the published version at https://doi.org/10.1192/bjo.2021.674. 


\section{Abstract}

Background: The term Orthorexia derives from the Greek "ortho - correct" and "orexis - appetite"; Orthorexia Nervosa is a pathological fixation with healthy eating that, starting with the idea to obtain a maximum health with a proper diet, leads to malnourishment and other medical sequelae, loss of relationships, loss of self-esteem, poor quality of life in general. Orthorexia, despite receiving broad empirical evidence, is not currently included in any psychiatric diagnostic manual. The main aim of this study is to investigate its presence in a sample of patients already diagnosed with a canonical eating disorder and also to understand eventual overlaps with other clinical disorders in order to optimize treatment and follow up. The ORTO-15 questionnaire, developed by an Italian team of researchers in 2005, was used to achieve the above aims: it is a tool comprehensive of 15 questions that assesses eating habits perceived as healthy. Really interesting and fascinating is to comprehend if people with a diagnosis of eating disorder present orthorectic behaviour and how this emerging reality fits in the Irish society with its peculiarities and uniqueness.

Results: The Point Prevalence obtained is $17.9 \%$. The expected rates of Orthorexia Nervosa in the general population are between $6.9 \%$ and $57.6 \%$, with a peak of $81.8 \%$ in specific populations, fact that places our examined sample in the lower side of the prevalence previously considered in other studies.

Conclusions: It is very difficult to comprehend and explain the reasons behind this fact and probably this is due to an overshadowing of symptoms with the major eating disorders. It is also significant the absence of correlation found between $\mathrm{OCD}$ and $\mathrm{ON}$ and also the fact that $\mathrm{ON}$ is more linked to Bulimia Nervosa in our sample rather than Anorexia Nervosa. The results obtained from this study give also a clear indication of the profile of the orthorexic patient, considered that the sample was obtained from a population of people with a diagnosis of Eating Disorder:

\section{Plain English Summary}

Orthorexia Nervosa is a fixation to healthy eating that can lead to severe physical consequences. Despite the fact that for many years several researchers from different countries came across this clinical entity, Orthorexia Nervosa at present is not included in any medical manual and is not formally defined. In this study we did investigate Anorexia Nervosa as clinical presence in the society, specifically in Irish society, and we also tried to outline the typical "Orthorexic Patient". The results obtained are in line with those obtained in other countries and also we did investigate the presence of Orthorexia Nervosa in conjunction with other illnesses such as Anorexia Nervosa and Obsessive Compulsive Disorder.

\section{Research Context}

The term Orthorexia Nervosa was coined in 1997 by American physician Steven Bratman in an article in Yoga Journal; he deepened the subject in 2000 in a book titled "Health Food Junkies" where he explores 
further the different aspects of ON, several types of extremely restrictive diets, the symptoms of ON, its progression, its diagnosis and finally suggestions regarding possible management.

Orthorexia nervosa diagnostic criteria proposed by Moroze et al:

Criterion A: Obsessional preoccupation with eating "healthy foods," focusing on concerns regarding the quality and composition of meals. (Two or more of the following.)

- Consuming a nutritionally unbalanced diet owing to preoccupying beliefs about food "purity."

- Preoccupation and worries about eating impure or unhealthy foods and of the effect of food quality and composition on physical or emotional health or both.

- Rigid avoidance of foods believed by the patient to be "unhealthy," which may include foods containing any fat, preservatives, food additives, animal products, or other ingredients considered by the subject to be unhealthy.

- For individuals who are not food professionals, excessive amounts of time (eg., 3 or more hours per day) spent reading about, acquiring, and preparing specific types of foods based on their perceived quality and composition.

- Guilty feelings and worries after transgressions in which "unhealthy" or "impure" foods are consumed.

- Intolerance to other's food beliefs.

- Spending excessive amounts of money relative to one's income on foods because of their perceived quality and composition.

Criterion $B$. The obsessional preoccupation becomes impairing by either of the following:

- Impairment of physical health owing to nutritional imbalances (eg., developing malnutrition because of an unbalanced diet).

- Severe distress or impairment of social, academic, or vocational functioning owing to obsessional thoughts and behaviours focusing on patient's beliefs about "healthy" eating.

Criterion $\boldsymbol{C}$. The disturbance is not merely an exacerbation of the symptoms of another disorder such as obsessive-compulsive disorder or of schizophrenia or another psychotic disorder.

Criterion $D$. The behaviour is not better accounted for by the exclusive observation of organized orthodox religious food observance or when concerns with specialized food requirements are in relation to professionally diagnosed food allergies or medical conditions requiring a special diet.

Really difficult is to establish connections between Orthorexia Nervosa and Obsessive Compulsive Disordersince they share fundamental traits such as intrusive thoughts, rituals regarding food preparation and fear of contamination deriving from contaminated aliments. Also challenging is to discriminate Orthorexia Nervosa from Anorexia Nervosa since once again they share important features 
such as focus on food quantity, fear of obesity, disturbed body image, drive for thinness and excessive exercising. According to Koven \& Abry's review, to complicate further the scenario, Orthorexia Nervosa, Obsessive Compulsive Disorder and Anorexia Nervosa are linked with the following characteristics:

- Perfectionism

- Cognitive rigidity

- Trait anxiety

- Impaired functioning

- Poor external monitoring

- Impaired working memory

Another matter to consider when examining Orthorexia Nervosa is obviously the lack of a clear and multidisciplinary management plan that could include, as suggested by some authors: physicians, psychotherapists, dieticians, psychiatrists, SSRIs, Olanzapine, exposure and response prevention, habit reversal training, etc.

Since 1997 many studies have been conducted in several countries with the aim to define clearer diagnostic criteria for $\mathrm{ON}$ and its prevalence and incidence in different populations such as students, body builders, general, psychiatric. It is necessary to understand if the phenomenon is present in Irish territory and what kind of relationship exists with the other eating disorders; the aim is to have a better understanding of the patient's clinical diagnosis to provide a better medical management and to ensure the best level of care possible.

On a broad research in PubMed emerged that in Ireland ON never inspired a particular research interest: the only traces and mentions of $\mathrm{ON}$ in Ireland refer to articles in The Irish Times, Indipendent.ie, a hint in the website Bodywhys.ie and minor references in other online sources, even if the phenomenon is known to psychiatrists and physicians all over the country.

\section{Research Questions}

Principal aim of the research is to establish the prevalence of Orthorexia Nervosa in a sample of patients attending Sligo/Leitrim Mental Health Services with a diagnosis of eating disorder, specifically those patients attending appointments with the eating disorder specialist assigned to the territory of Sligo, Leitrim, West Cavan and South Donegal and referred by their psychiatric treating teams.

\section{Secondary aims.}

- Observe any connection between $\mathrm{ON}$ and age, gender, race, education, employment.

- Observe if healthy eating reflects in broader healthy lifestyle habits as abstinence from alcohol and smoking and increase in physical exercise.

- Observe if there is any connection between vegetarian and/or vegan diet and ON. 
- Observe correlation between BMI and ON when applicable.

- Observe relationship between $\mathrm{ON}$ and other eating disorders.

- Observe comorbidity with other psychiatric illnesses.

\section{Research Methods}

Cross-sectional observational study aimed to establish the prevalence of Orthorexia Nervosa in a population of patients who have already received a diagnosis of an eating disorder by treating consultants and who are actively attending appointments with an eating disorder nurse specialist in the above mentioned territory covered by Sligo - Leitrim Mental Health Services. Correlations between orthorexic behaviours and data present in the demographic grid were made and appropriate inferences were also made to recognize eventual trends in age, sex, education, employment, psychiatric diagnosis, etc.

Sampling: Patients attending appointments with the eating disorder nurse specialist appointed to Sligo Leitrim Mental Health Services were approached at the time of the appointment: the aim of the study was orally explained and written consent to participate the study was also obtained.

Inclusion criteria: age between 18 and 65 years; to have a diagnosis of eating disorder (ICD10 code); to have met the eating disorder nurse specialist at least once in the last 5 years for a clinical appointment.

Exclusion criteria: age below 18 years and above 65; not having a diagnosis of an eating disorder according to ICD10 criteria; did not meet the eating disorder nurse specialist for a clinical appointment in the last 5 years.

Tests: Every patient was asked to complete a demographic grid (elaborated by the researchers, which includes information regarding: age, gender, race, weight, height, hours of weekly exercise, years of education, employment situation, medical illnesses, smoking habits, type of diet, average weekly alcohol intake) and the Orto-15 questionnaire. The Orto-15 questionnaire is a tool produced by Donini et al. in Italy in 2005 with the view to diagnose $\mathrm{ON}$ : it is a 15 questions self-administered questionnaire that assesses highly sensitive behaviours linked to healthy and proper nutrition and that explore the person's attitude towards selection, purchase, preparation, consumption of the food and also feelings, emotions and obsessions related to it. Every question is answered in a four-point Likert scale, where 1 is indicative of an orthorexic behaviour and 4 is linked to normal eating behaviour; scores below 40 points are diagnostic of Orthorexia Nervosa according to the developers of the test. At the threshold of 40 points, the Orto- 15 test presents the following values: sensitivity $100 \%$, specificity $73.6 \%$, positive predictive value $17.6 \%$, negative predictive value $100 \%$. For every patient, the staff member collecting the data did indicate in a separate grid the primary eating disorder of the patient (with ICD-10 code), eventual comorbid psychiatric illnesses (with ICD-10 codes) and BMI if possible; these information were obtained from the clinical chart of the patient. The Orto-15 test, the demographic grid and the staff grid were completely anonymous, the names of the patients contacted were not recorded anywhere and were not associated 
with their completed tests. Orto-15 questionnaire was scored by one of the researchers in a different occasion. Data were stored in one of the researchers locked office.

Procedure: Patients were contacted by phone by the researchers and an appointment was offered where the study was explained, the consent was signed and the demographic grid and the Orto - 15 Questionnaire were completed. In case the patient did express the desire to participate the study but not willing to attend an appointment, information about the study was given by phone and the consent form, the demographic grid and the Orto - 15 Questionnaire have been sent by post (the patient will post them back to the researchers). If the patient contacted did not agree to take part to the study, no further contact in regards to it was made. Weight, Height and BMI were obtained only from the patients who did attend an appointment.

Consent forms were not paired with demographic grids and Orto - 15 Questionnaire, hence an acceptable level of blinding was obtained.

The participants, their General Practitioners and their treating Psychiatric teams were not informed of the score obtained in the Orto - 15 Questionnaire, since the purpose of this research is descriptive only.

The collected data were transferred in a Microsoft Excel file and the hard copies were stored in a locked cabinet in one of the researchers' offices. Data were analysed as explained below.

Data analysis: For the patients who scored below 40 at the Orto-15 questionnaire were calculated range, mean, median, mode, variance and standard deviation. Prevalence of ON was calculated and also percentages of the different ICD-10 diagnosis.

Significance of Research: Researching on the field of Orthorexia Nervosa is innovative, interesting and truly fascinating. It constitutes an opportunity to understand more of this phenomenon in an Irish sample and also it is a great opportunity to open new research scenarios with the final purpose to improve care planning for patients with Eating Disorders. At present, by treating Anorexia, Bulimia or Binge Eating Disorder we hope to treat also some behaviours here defined as "orthorexic" without aiming to treat them; investigating more about $\mathrm{ON}$ we will have the chance to redefine our clinical practice and to tailor our services to suit patients with different needs and personality traits. By understanding more about ON, we will understand more about our patients, how their primary psychiatric illnesses interact with $\mathrm{ON}$ and we will also understand more about our society and its trends regarding food habits.

\section{Discussion}

128 questionnaires were handed or posted to the patients attending the Eating Disorders Nurse Specialist as above indicated: 5 people were no longer at their addresses, 92 people refused to participate or never posted the questionnaires back, 31 people responded. Of the 31 questionnaires received, 2 were incomplete, 1 was excluded because the respondent was 17 years old and 29 were complete and compliant with the proposed exclusion and inclusion criteria, of them 23 were positive for diagnosis of 
Orthorexia and 5 negative. All the following inferences are based on the 23 positive Ortho-15 questionnaires obtained. The Point Prevalence obtained is $17.9 \%$. Regarding the age, the range is from 18 years old to 60 years old, with a mean of 37.7, a median of 39 and a mode of 18 . All the people who scored positively were women and 22 over 23 (95.7\%) were Caucasians. Regarding the hours of weekly exercise, the obtained range was from 0 to 14 hours, with a mean of 4.6 hours per week; 6 over 23 spaces $(26.1 \%)$ were left vacant by the responders. Regarding years of education, the obtained reange varied from 6 to 17 years with a mean of 14.1 years of education; 4 over 23 spaces (17.4\%) were left vacant. Regarding employment the results obtained show a wide variety: 3 over 23 vacant spaces (13\%), 6 unemployed, 3 students, 2 retired, 2 shop/sales assistants, 2 teachers, 1 pharmacist, 1 "family support", 1 person "on sick leave", 1 care assistant, 1 "self-employed". Regarding comorbid illnesses a wide list was reported, including general medical and psychiatric symptoms and signs, with poor certainty regarding conditions diagnosed by GP or other medical professionals. The reported psychiatric difficulties also did not match their official ICD10 comorbid diagnosis; 5 over 23 spaces $(21.7 \%)$ were left vacant. Regarding smoking status, 5 over 23 positive responders were smokers $(21.7 \%)$ and 18 non-smokers $(78.3 \%)$. Regarding eating habits, 19 over 23 responders had a standard eating profile (82.6\%), 2 vegetarians, 1 vegan and 1 vacant space. Regarding alcohol consumption, only 1 responder reported consuming 16 units of alcohol per week (4\%), 4 people consuming between 1 and 10 units of alcohol per week (17.4\%), 17 not drinking alcohol (74\%) and 1 space left vacant. Regarding the diagnosis of Eating Disorders, 9 people (39.1\%) had a diagnosis of Bulimia Nervosa (ICD10 code F50.2), 6 positive responders $(26.1 \%)$ had a diagnosis of Anorexia Nervosa (F50.0), 3 people Overeating associated with other psychological disturbances, 2 Atypical Bulimia Nervosa and 2 Eating Disorder, unspecified. All the positive responders did not present any other diagnosed psychiatric illness, even though several psychiatric symptoms were reported in the forms received, such as "anxiety, panic attacks, depression, sadness, etc". BMI ranged from 14.4 to 45.8 , with a mean of 24.9 , a median of 23.3 and a mode of 20.2; mean, median and mode are included in Normal Range according to the WHO BMI Classification. Orto-15 score ranged between 27 and 39 points.

\section{Conclusions}

The results obtained from this study give a clear indication of the profile of the orthorexic patient, considered that the sample was obtained from a population of people with a diagnosis of Eating Disorder:

- Caucasian woman in her 30s

- Exercising 5 hours per week

- Secondary education

- Unemployed

- Non-smoker

- Diagnosis of Anorexia Nervosa

- No other comorbid psychiatric illnesses 
- Standard pattern of eating

- Minimal or absent alcohol consumption

- Normal range BMI

According to previous Italian studies (Ramacciotti et al. 2011), the expected rates of Orthorexia Nervosa in the general population are between $6.9 \%$ and $57.6 \%$, with a peak of $81.8 \%$ in specific populations, fact that places our examined sample in the lower side of the prevalence previously considered. It is very difficult to comprehend and explain the reasons behind this fact and probably this is due to an overshadowing of symptoms with the major eating disorders. It is also significant the absence of correlation found between $\mathrm{OCD}$ and $\mathrm{ON}$ and also the fact that $\mathrm{ON}$ is more linked to Bulimia Nervosa in our sample rather then Anorexia Nervosa.

\section{List Of Abbreviations}

ON: Orthorexia Nervosa

OCD: Obsessive Compulsive Disorder

BMI: Body Mass Index

ICD-10: International Classification of Diseases (10th revision)

GP: General Practitioner

\section{Limitations}

we recognised a limited number of limitations in our study:

- Limited number of responders: 128 people contacted because suiting the inclusion criteria and only 29 responded ( 1 excluded because below 18 years of age), so only the $21.7 \%$ of the sample considered was actually scored.

- Age limitations (only 18 - 65 group considered)

- All the sample was composed by women

- No psychiatric comorbidities in the sample

- No specifications obtained regarding diagnosed medical illnesses

\section{Future Research}

This study establishes a base to conduct further research in an Irish contest. From our point of view, this study could be extended in the future to CAMHS and Psychiatry of Old Age population, in order to understand if there are any differences with the sample we examined and also to have a broader base for future studies. Obviously a big goal would be to apply this protocol to a sample taken from the entire 
population, but we understand that this would be a great difficulty in terms of management of the study itself, funding, workforce.

\section{Declarations}

Ethical Approval and Consent to Participate: Ethical Approval obtained from Sligo University Hospital Research Ethics Committee. Written Consent to Participate obtained from every individual involved in the research.

Consent for Publication: Consent for publication given from all the authors involved in the research.

Availability of Supporting Data: Supporting data fully available in papery and digital format.

Competing Interests: The authors do not have any competing interests to declare for this study

Funding: No funding was available for this study

\section{Authors' Contributions:}

- Dr Ignazio Graffeo: protocol elaboration, ethical approval, data analysis, article writing

- Mary Harron: data collection, protocol elaboration

- Dr Edmond O’Mahony: protocol elaboration, supervision.

Acknowledgements: Not applicable

\section{References}

1. Alvarenga, M. S., Martins, M. C. T., Sato, K. S. C. J., Vargas, V.A., Philippi, S.T. and Scagliusi, F.B. (2012) "Orthorexia nervosa behaviour in a sample of Brazilian dieticians assessed by the Portuguese version of ORTO-15", Eating Weight Disorder, 17, pp. 29-35.

2. Bo, S., Zoccali, R., Ponzo, V., Soldati, L., De Carli, L., Benso, A., Fea, E., Rainoldi, A., Durazzo, M., Fassino, S. and Abbate-Daga, G. (2008) "University courses, eating problems and muscle dysmorphia: are there any associations?", Journal of Translational Medicine, 12, pp. 221.

3. Bratman, S. (2017) "Orthorexia vs. theories of healthy eating", Eating Weight Disorder, 22, pp. 381385.

4. Bratman, S. and Knight, D. (2000)"Health Food Junkies: Orthorexia Nervosa- Overcoming the Obsession With Healthful Eating", Journal of Translational Medicine, 10, pp. 218.

5. Brytek-Matera, A., Krupa, M., Poggiogalle. E. and Donini, L. M. (2014) "Adaptation of the ORTHO-15 test to Polish women and men", Eating Weight Disorder, 19, 69-76.

6. Brytek-Matera, A., Donini, L. M., Krupa, M., Poggiogalle, E. and Hay, P. (2016) "Erratum to: Orthorexia nervosa and self-attitudinal aspects of body image in female and male university students", Journal of Eating Disorder,4-16. 
7. Brytek-Matera, A., Rogoza, R., Gramaglia, C. and Zeppegno, P. (2015) “Predictors of orthorexic behaviours in patients with eating disorders: a preliminary study", BMC Psychiatry, 15, pp. 252.

8. Cuzzolaro, M. and Donini, L. M. (2016) “Orthorexia nervosa by proxy?”, Eating Weight Disorder , 21, pp.549-551.

9. Dell'osso, L., Abelli, M., Carpita, B., Massimetti, G., Pini, S., Rivetti, L., Gorrasi, F., Tognetti, R., Ricca, V. and Carmassi, C. (2017) "Orthorexia nervosa in a sample of Italian university population", Riv Psichiatr, 51(5), pp. 190-196.

10. Dell'osso, L., Abelli, M., Carpita, B., Pini, G., Castellini, G., Carmassi, C. and Ricca, A. (2016) "Historical evolution of the concept of anorexia nervosa and relationships with orthorexia nervosa, autism, and obsessive-compulsive spectrum", Neuropsychiatric Disease and Treatment, pp. 1650-1660.

11. Depa, J., Schweizer, J., Bekers, S. K., Hilzendegen, C. and Stroebele-Benschop, N. (2016) "Prevalence and predictors of orthorexia nervosa among German students using the 21-item-DOS", Department of Nutritional Psychology, pp. 16-19.

12. Donini, L. M., Marsili, D., Graziani, M. P., Imbriale, M. and Cannella. C. (2005) “Orthorexia nervosa: Validation of a diagnosis questionnaire", Eating Weight Disorder, 10 (1), pp. 28-32.

13. Dunn, T. M. and Bratman, S. (2015) "On orthorexia nervosa: A review of the literature and proposed diagnostic criteria”, Eating Behaviours, 21, pp. 11-17.

14. Eriksson, E., Baigi, A., Marklund, B. and Lindgren, E. C. (2008) "Social physique anxiety and sociocultural attitudes toward appearance impact on orthorexia test in fitness participants", Scand $J$ Med Sci Sports, 18, pp. 389-394.

15. Gramaglia, C., Brytek-Matera, A., Rogoza, R. and Zeppegno, P. (2017) “Orthorexia and anorexia nervosa: two distinct phenomena? A cross-cultural comparison of orthorexic behaviours in clinical and non-clinical samples", BMC Psychiatry, 17, pp. 75.

16. Håman, L., Lindgren, E. C. \& Prell, H. (2017) “If it's not Iron its Iron f*cking biggest Ironman”: personal trainers' views on health norms, orthorexia and deviant behaviours", International Journal of Qualitative Studies on Health and wellbeing, 12, pp. 1-10.

17. Haman, L., Barker-Rutchi, N., Patriksson, G. and Lindgren, E. C. (2015) "Orthorexia nervosa: An integrative literature review of a lifestyle syndrome", International Journal of Qualitative Studies on Health and Well-being, 10, pp.1-10.

18. Hyrnik, J., Janas-Kozik, M., Stochel, M., Jelonek, I., Siwiec, A. and Rybakowski, J. K. (2016) "The Assessment of Orthorexia Nervosa among 1899 Polish Adolescents Using the ORTO-15 Questionnaire", International Journal of Psychiatry in Clinical Practice, 20 (3), pp. 199-203.

19. Korinth, A., Schiess, S. \& Westenhoefer, J. (2009) "Eating behaviour and eating disorders in students of nutrition sciences", Public Health Nutrition, 13(1), pp. 32-37.

20. Koven, N. S. and Abry, A. W. (2015) "The Clinical Basis of Orthorexia Nervosa: Emerging Perspectives", Neuropsychiatric Disease and Treatment, 11, pp. 385-394.

21. Kummer, A., Dias, F. M. V. and Teixeira, A. L. (2008) "On the concept of orthorexia nervosa", Scand J Med Sci Sports, 18, pp. 395-396. 
22. Michalska, A., Szejko, N., Jakubczyk, A. and Wojnar, M. (2016) "Nonspecific eating disorders: a subjective review", Pol, 50(3), pp. 497-507.

23. Missbach, B. and Barthels, F. (2017) "Editorial Orthorexia Nervosa: moving forward in the field", Eating Weight Disorder, 22(1).

24. Missbach, B., Hinterbuchinger, B., Dreiseitl, V., Zellhofer, S., Kurz, C. and König, J. (2015) "When Eating Right, Is Measured Wrong! A Validation and Critical Examination of the ORTO-15 Questionnaire in German", Plos One, 1, pp. 1-4.

25. Nevin, S. M. and Vartanian, L. R. (2017) "The stigma of clean dieting and orthorexia", Journal of Eating Disorders, 5 (37), pp. 1-10.

26. Nevin, S., Emine, Y., Saniye, B., Gulsah, S. \& Bülent, C. (2016) "Does the rise in eating disorders lead to increasing risk of orthorexia nervosa? Correlations with gender, education, and body mass index", Ecology of Food and Nutrition, pp. 55-3.

27. Olejniczak, D., Bugajec, D., Panczyk, M., Brytek-Matera, A., Religioni, U., Czerw, A., Grąbczewska, A., Juszczyk, G., Jabłkowska-Górecka, K. and Staniszewska, A. (2017) "Analysis concerning nutritional behaviours in the context of the risk of orthorexia", Neuropsychiatric Disease and Treatment, 13, pp. 543-550.

28. Sang Won, P., Jeong, Yup, K., Gang Ji, G., Eun Sil, J., Heui Jung, P. and Young Joo, K. (2011) "Orthorexia Nervosa with Hyponatremia, Subcutaneous Emphysema, Pneumomediastimum, Pneumothorax and Pancytopenia", Electrolyte Blood Press, 9, pp. 32-37.

29. Stochel, M., Janas-Kozik, M., Zejda, E., Hyrnik, J., Jelonek, I. and Siwiec, A. (2015) "Validation of ORTO-15 Questionnaire in the group of urban youth aged 15-21", Pol, 49(1), pp. 119-134.

30. Turner, P. G. and Lefevre, C. E. (2017) "Instagram use is linked to increased symptoms of orthorexia nervosa”, Eat Weight Disorder, (22) pp. 277-284.

31. Varga, M., Konkolÿ Thege, B., Dukay-Szabó, S., Túry, F. and van Furth, E. F. (2014) “When eating healthy is not healthy: orthorexia nervosa and its measurement with the ORTO-15 in Hungary", BMC Psychiatry, 14, pp. 59. 